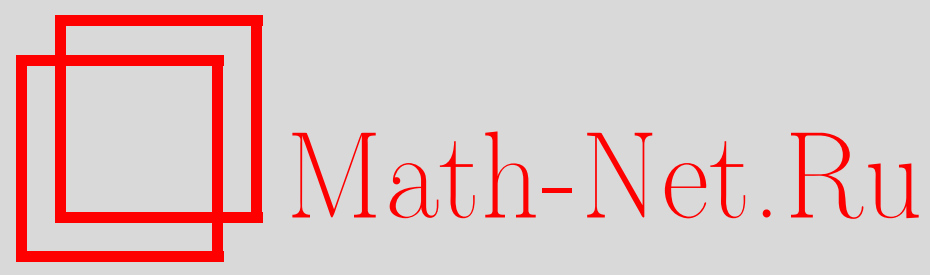

В. А. Краснов, О числах Пикара и Лефшеца вещественной алгебраической поверхности, Матем. заметки, 1998, том 63, выпуск 6, 847-852

DOI: https://doi.org/10.4213/mzm1354

Использование Общероссийского математического портала Math-Net.Ru подразумевает, что вы прочитали и согласны с пользовательским соглашением http://www . mathnet.ru/rus/agreement

Параметры загрузки:

IP : 54.80 .97 .219

26 апреля 2023 г., 18:11:55

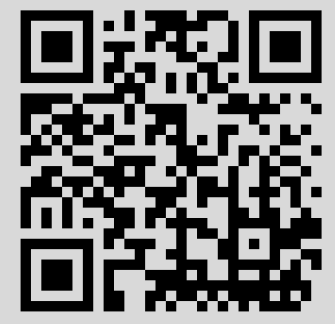




\section{О ЧИСЛАХ ПИКАРА И ЛЕФШЕЦА ВЕЩЕСТВЕННОЙ АЛГЕБРАИЧЕСКОЙ ПОВЕРХНОСТИ}

\section{В. А. Краснов}

Определяются два числа Пикара и два числа Лефшеца для вещественной алгебраической поверхности. Они аналогичны числу Пикара и числу Лефшеца для комплексной алгебраической поверхности. Для этих чисел доказьваются оценки и соотношения в виде неравенств.

Библиография: 6 названий.

Пусть $X$ - неособая вещественная проективная поверхность. Для комплексной поверхности $X_{\mathbb{C}}=X \otimes \mathbb{C}$ число Пикара $\rho\left(X_{\mathbb{C}}\right)$ и число Лефшшеца $\rho_{0}\left(X_{\mathbb{C}}\right)$ определяются следуюшим образом. Если

$$
\operatorname{cl}_{\mathbb{C}}: \operatorname{Div} X_{\mathbb{C}} \rightarrow H^{2}(X(\mathbb{C}), \mathbb{Z})
$$

- отображение цикла, то образ этого отображения обозначается через $H_{\mathrm{alg}}^{2}(X(\mathbb{C}), \mathbb{Z})$, коядро - через $H_{\mathrm{tr}}^{2}(X(\mathbb{C}), \mathbb{Z})$, а их ранги - через $\rho\left(X_{\mathbb{C}}\right), \rho_{0}\left(X_{\mathbb{C}}\right)$ соответственно. Для вещественной поверхности $X$ определены также следующие отображения цикла (см. [1]):

$$
\text { cl: } \operatorname{Div} X \rightarrow H^{2}\left(X(\mathbb{C}) ; G, \mathbb{Z}_{-}\right), \quad \operatorname{cl}_{\mathbb{R}}: \operatorname{Div} X \rightarrow H^{1}\left(X(\mathbb{R}), \mathbb{F}_{2}\right),
$$

где $G=G(\mathbb{C} \backslash \mathbb{R})$ - группа Галуа, $\mathbb{Z}_{-}-G$-модуль $\mathbb{Z}$, на котором образующая $g \in G$ действует умножением на -1 . Тогда мы можем определить алгебраические группы когомологий $H_{\mathrm{alg}}^{2}\left(X(\mathbb{C}) ; G, \mathbb{Z}_{-}\right), H_{\mathrm{alg}}^{1}\left(X(\mathbb{R}), \mathbb{F}_{2}\right)$ как образы отображений цикла $\mathrm{cl}, \mathrm{cl} \mathbb{R}$, a также трансцендентные группы когомологий $H_{\mathrm{tr}}^{2}\left(X(\mathbb{C}) ; G, \mathbb{Z}_{-}\right), H_{\mathrm{tr}}^{1}\left(X(\mathbb{R}), \mathbb{F}_{2}\right)$ как коядра отображений $\mathrm{cl}, \mathrm{cl}_{\mathbb{R}}$. Ранги групп $H_{\mathrm{alg}}^{2}\left(X(\mathbb{C}) ; G, \mathbb{Z}_{-}\right), H_{\mathrm{tr}}^{2}\left(X(\mathbb{C}) ; G, \mathbb{Z}_{-}\right)$обозначим через $\rho(X), \rho_{0}(X)$, а размерности пространств $H_{\mathrm{alg}}^{1}\left(X(\mathbb{R}), \mathbb{F}_{2}\right), H_{\mathrm{tr}}^{1}\left(X(\mathbb{R}), \mathbb{F}_{2}\right)$ - через $\rho(X(\mathbb{R})), \rho_{0}(X(\mathbb{R}))$.

ПРЕДЛОЖЕНИЕ. Выполняются неравенства

$$
\begin{gathered}
\rho(X) \leqslant \frac{1}{2}\left(h^{1,1}(X(\mathbb{C}))-\chi(X(\mathbb{R}))\right)+1, \\
0 \leqslant \rho\left(X_{\mathbb{C}}\right)-\rho(X) \leqslant \frac{1}{2}\left(h^{1,1}(X(\mathbb{C}))+\chi(X(\mathbb{R}))\right)-1, \\
h^{2,0}(X(\mathbb{C})) \leqslant \rho_{0}\left(X_{\mathbb{C}}\right)-\rho_{0}(X) \leqslant h^{2,0}(X(\mathbb{C}))+\frac{1}{2}\left(h^{1,1}(X(\mathbb{C}))+\chi(X(\mathbb{R}))\right)-1,
\end{gathered}
$$

где $h^{1,1}(X(\mathbb{C})), h^{2,0}(X(\mathbb{C}))$ - числа Ходжа, $\chi(X(\mathbb{R}))$ - эйлерова характеристика. 
ДокАЗАТЕЛЬСтво. Пусть $\operatorname{Div}_{h} X_{\mathbb{C}}-$ группа дивизоров гомологичных нулю. Тогда имеем точную последовательность $G$-модулей

$$
0 \rightarrow \operatorname{Div}_{h} X_{\mathbb{C}} \rightarrow \operatorname{Div} X_{\mathbb{C}} \stackrel{\mathrm{cl}_{\mathbb{C}}}{\longrightarrow} H_{\mathrm{alg}}^{2}\left(X(\mathbb{C}), \mathbb{Z}_{-}\right) \rightarrow 0
$$

Из нее получаем длинную точную последовательность

$$
0 \rightarrow\left(\operatorname{Div}_{h} X_{\mathbb{C}}\right)^{G} \rightarrow\left(\operatorname{Div} X_{\mathbb{C}}\right)^{G} \stackrel{\mathrm{cl}_{\mathbb{C}}}{\longrightarrow} H_{\mathrm{alg}}^{2}\left(X(\mathbb{C}), \mathbb{Z}_{-}\right)^{G} \rightarrow H^{1}\left(G, \operatorname{Div}_{h} X_{\mathbb{C}}\right) \rightarrow \cdots
$$

Так как $H^{1}\left(G, \operatorname{Div}_{h} X_{\mathbb{C}}\right)$ - 2-элементарная групша, т.е. является $\mathbb{F}_{2}$-пространством, то из полученной точной последовательности следует равенство

$$
\operatorname{rk}\left(\operatorname{cl}_{\mathbb{C}}\left(\left(\operatorname{Div} X_{\mathbb{C}}\right)^{G}\right)\right)=\operatorname{rk} H_{\text {alg }}^{2}\left(X(\mathbb{C}), \mathbb{Z}_{-}\right)^{G}
$$

Рассмотрим коммутативную диаграмму

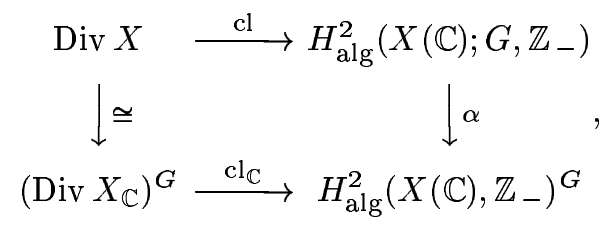

где $\alpha$ - гомоморфизм забывания вещественной структуры

$$
H^{2}\left(X(\mathbb{C}) ; G, \mathbb{Z}_{-}\right) \rightarrow H^{2}\left(X(\mathbb{C}), \mathbb{Z}_{-}\right)
$$

его ядро является 2-элементарной группой (см. [2]), поэтому имеем равенства

$$
\rho(X)=\operatorname{rk} H_{\mathrm{alg}}^{2}\left(X(\mathbb{C}) ; G, \mathbb{Z}_{-}\right)=\operatorname{rk}\left(\operatorname{cl}_{\mathbb{C}}\left(\left(\operatorname{Div} X_{\mathbb{C}}\right)^{G}\right)\right)
$$

Из (4), (5) следуют равенства

$$
\rho(X)=\operatorname{rk} H_{\text {alg }}^{2}\left(X(\mathbb{C}), \mathbb{Z}_{-}\right)^{G}=\operatorname{rk}\left(H_{\text {alg }}^{2}\left(X(\mathbb{C}), \mathbb{Z}_{-}\right) / \text {Tors }\right)^{G} .
$$

Положим $H=H^{2}(X(\mathbb{C}), \mathbb{Z}) /$ Tors. Тогда имеет место каноническое вложение $H \subset$ $H^{2}(X(\mathbb{C}), \mathbb{C})$, а теорема Лефшеца об $(1,1)$-классах дает равенство

$$
H_{\text {alg }}^{2}(X(\mathbb{C}), \mathbb{Z}) / \text { Tors }=H \cap H^{1}(X(\mathbb{C}))
$$

Пусть $H_{+}, H_{-}-$соответственно инвариантная и антиинвариантная относительно $g$ подгруппы групшы $H$. Тогда из (6), (7) получаем равенство

$$
\rho(X)=\operatorname{rk}\left(H_{-} \cap H^{1,1}(X(\mathbb{C}))\right) .
$$

Пусть $H^{1,1}(X(\mathbb{C}))_{+}, H^{1,1}(X(\mathbb{C}))_{-}$- соответственно инвариантное и антиинвариантное относительно $g$ подпространства пространства $H^{1,1}(X(\mathbb{C}))$, a $h_{+}^{1,1}, h_{-}^{1,1}$ - их размерности. Тогда из равенства (8) следует неравенство

$$
\rho(X) \leqslant h_{-}^{1,1} .
$$


$\mathrm{C}$ другой стороны, из формулы Лефшеца о неподвижных точках инволюции $g: X(\mathbb{C}) \rightarrow$ $X(\mathbb{C})$ следует равенство (см. [3])

$$
\chi(X(\mathbb{R}))=2+h^{1,1}(X(\mathbb{C}))-2 h_{-}^{1,1},
$$

поэтому неравенства (9) и (1) равносильны. Так как

$$
\rho\left(X_{\mathbb{C}}\right)=\operatorname{rk}\left(H \cap H^{1,1}(X(\mathbb{C}))\right),
$$

из равенства (8) получаем неравенства

$$
0 \leqslant \rho\left(X_{\mathbb{C}}\right)-\rho(X) \leqslant h_{+}^{1,1} .
$$

Равенство (10) можно переписать в виде

$$
\chi(X(\mathbb{R}))=2+2 h_{+}^{1,1}-h^{1,1}(X(\mathbb{C})),
$$

поэтому неравенства (11) и (2) равносильны. Осталось доказать неравенства (3). Так как

$$
\begin{aligned}
\rho_{0}\left(X_{\mathbb{C}}\right) & =\operatorname{rk} H^{2}(X(\mathbb{C}), \mathbb{Z})-\rho\left(X_{\mathbb{C}}\right)=2 h^{2,0}(X(\mathbb{C}))+h^{1,1}(X(\mathbb{C}))-\rho\left(X_{\mathbb{C}}\right) \\
\rho_{0}(X) & =\operatorname{rk} H^{2}\left(X(\mathbb{C}) ; G, \mathbb{Z}_{-}\right)-\rho(X)=\operatorname{rk} H^{2}\left(X(\mathbb{C}), \mathbb{Z}_{-}\right)^{G}-\rho(X) \\
& =h^{2,0}(X(\mathbb{C}))+h_{-}^{1,1}-\rho(X),
\end{aligned}
$$

имеем

$$
\rho_{0}\left(X_{\mathbb{C}}\right)-\rho_{0}(X)=h^{2,0}(X(\mathbb{C}))+h_{+}^{1,1}-\left(\rho\left(X_{\mathbb{C}}\right)-\rho(X)\right) .
$$

Из этого равенства и неравенств (11) следуют неравенства

$$
h^{2,0}(X(\mathbb{C})) \leqslant \rho_{0}\left(X_{\mathbb{C}}\right)-\rho_{0}(X) \leqslant h^{2,0}(X(\mathbb{C}))+h_{+}^{1,1},
$$

которые равносильны неравенствам (3). Предложение доказано.

Теорема. Пусть $X(\mathbb{R}) \neq \varnothing$. Тогда справедливы утверждения:

1) если группа $H^{*}(X(\mathbb{C}), \mathbb{Z})$ свободная, то выполняется неравенство

$$
\rho(X(\mathbb{R})) \leqslant \rho(X)+q(X),
$$

которое становится равенством для $M$-поверхности, где $q(X)$ - иррегулярность;

2) если $H^{1}(X(\mathbb{C}), \mathbb{Z})=0$, то для $(M-k)$-поверхности $X$ выполняются неравенства

$$
\begin{gathered}
\rho(X)-k \leqslant \rho(X(\mathbb{R})) \leqslant \rho(X), \\
\rho_{0}(X)-k \leqslant \rho_{0}(X(\mathbb{R})) \leqslant \rho_{0}(X) .
\end{gathered}
$$


ДокАЗАТЕЛЬСтво. Рассмотрим отображение ограничения эквивариантных когомологий

$$
\beta: H^{2}\left(X(\mathbb{C}) ; G, \mathbb{Z}_{-}\right) \rightarrow H^{2}\left(X(\mathbb{R}) ; G, \mathbb{Z}_{-}\right) .
$$

Так как $H^{2}\left(X(\mathbb{R}) ; G, \mathbb{Z}_{-}\right)=H^{1}\left(X(\mathbb{R}), \mathbb{F}_{2}\right)$ (см. [4]), получаем гомоморфизм

$$
\beta: H_{\mathrm{alg}}^{2}\left(X(\mathbb{C}) ; G, \mathbb{Z}_{-}\right) \rightarrow H^{1}\left(X(\mathbb{R}), \mathbb{F}_{2}\right)
$$

В [4] установлено, что этот гомоморфизм удовлетворяет условию $\beta$ о $\mathrm{cl}=\mathrm{cl}_{\mathbb{R}}$. Таким образом, имеем равенство

$$
H_{\mathrm{alg}}^{1}\left(X(\mathbb{R}), \mathbb{F}_{2}\right)=\beta\left(H_{\mathrm{alg}}^{2}\left(X(\mathbb{C}) ; G, \mathbb{Z}_{-}\right)\right)
$$

Следовательно,

$$
\begin{aligned}
\rho(X(\mathbb{R})) & =\operatorname{dim} \beta\left(H_{\mathrm{alg}}^{2}\left(X(\mathbb{C}) ; G, \mathbb{Z}_{-}\right)\right)=\operatorname{dim} \beta\left(H_{\mathrm{alg}}^{2}\left(X(\mathbb{C}) ; G, \mathbb{Z}_{-}\right) \otimes \mathbb{F}_{2}\right) \\
& \leqslant \operatorname{dim} H_{\mathrm{alg}}^{2}\left(X(\mathbb{C}) ; G, \mathbb{Z}_{-}\right) \otimes \mathbb{F}_{2},
\end{aligned}
$$

причем для $M$-поверхности последнее неравенство становится равенством, так как для нее гомоморфизм

$$
\beta: H^{2}\left(X(\mathbb{C}) ; G, \mathbb{Z}_{-}\right) \otimes \mathbb{F}_{2} \rightarrow H^{1}\left(X(\mathbb{R}), \mathbb{F}_{2}\right)
$$

является мономорфизмом (см. [1]). Покажем, что в случае, когда группа $H^{*}(X(\mathbb{C}), \mathbb{Z})$ свободная, вьполняется неравенство

$$
\operatorname{dim} H_{\mathrm{alg}}^{2}\left(X(\mathbb{C}) ; G, \mathbb{Z}_{-}\right) \otimes \mathbb{F}_{2} \leqslant \rho(X)+q(X),
$$

причем для $M$-поверхности оно становится равенством. Рассмотрим точную последовательность $G$-пучков на $X(\mathbb{C})$

$$
0 \rightarrow \mathbb{Z}_{-} \stackrel{2 \pi i}{\longrightarrow} \mathscr{O} \stackrel{\exp }{\longrightarrow} \mathscr{O}^{*} \rightarrow 1
$$

где образующая $g \in G$ действует на $\mathscr{O}, \mathscr{O}^{*}$ по правилу: $\left(g^{*}(h)\right)(x)=\overline{h(g(x))}$ (черта означает комплексное сопряжение). Из (15) получаем длинную точную последовательность эквивариантных когомологий

$$
\cdots \rightarrow H^{1}\left(X(\mathbb{C}) ; G, \mathscr{O}^{*}\right) \stackrel{\delta}{\rightarrow} H^{2}\left(X(\mathbb{C}) ; G, \mathbb{Z}_{-}\right) \rightarrow H^{2}(X(\mathbb{C}) ; G, \mathscr{O}) \rightarrow \cdots
$$

Образ связывающего гомоморфизма $\delta$ в ней равен группе $H_{\mathrm{alg}}^{2}\left(X(\mathbb{C}) ; G, \mathbb{Z}_{-}\right)($см. $[1])$. Так как группа $H^{2}(X(\mathbb{C}) ; G, \mathscr{O})=H^{2}(X(\mathbb{C}), \mathscr{O})^{G}$ не имеет кручения, группа кручения $H^{2}(X(\mathbb{C}) ; G, \mathbb{Z})_{\text {tors }}$ всегда содержится в групе $H_{\text {alg }}^{2}(X(\mathbb{C}) ; G, \mathbb{Z}-)$. Так как група $H^{2}(X(\mathbb{C}), \mathbb{Z})$ свободная, групша кручения $H^{2}(X(\mathbb{C}) ; G, \mathbb{Z}-)_{\text {tors }}$ равна ядру гомоморфизма забывания вешественной структуры

$$
\alpha: H^{2}\left(X(\mathbb{C}) ; G, \mathbb{Z}_{-}\right) \rightarrow H^{2}\left(X(\mathbb{C}), \mathbb{Z}_{-}\right)
$$


Ядро гомоморфизма $\alpha$ равно 2-элементарной групше $H^{1}\left(G, H^{1}\left(X(\mathbb{C}), \mathbb{Z}_{-}\right)\right)$, что вытекает из рассмотрения спектральной последовательности (см. [2])

$$
\mathrm{II}_{2}^{p, q}=H^{p}\left(G, H^{q}\left(X(\mathbb{C}), \mathbb{Z}_{-}\right)\right) \Longrightarrow H^{p+q}\left(X(\mathbb{C}) ; G, \mathbb{Z}_{-}\right) .
$$

Таким образом, имеем точную последовательность груш

$$
0 \rightarrow H^{1}\left(G, H^{1}\left(X(\mathbb{C}), \mathbb{Z}_{-}\right)\right) \rightarrow H_{\mathrm{alg}}^{2}\left(X(\mathbb{C}) ; G, \mathbb{Z}_{-}\right) \rightarrow \mathbb{Z}^{\rho(X)} \rightarrow 0 .
$$

Отсюда следует равенство

$$
\operatorname{dim} H_{\mathrm{alg}}^{2}\left(X(\mathbb{C}) ; G, \mathbb{Z}_{-}\right) \otimes \mathbb{F}_{2}=\rho(X)+\operatorname{dim} H^{1}\left(G, H^{1}\left(X(\mathbb{C}), \mathbb{Z}_{-}\right)\right) .
$$

Осталось заметить, что всегда

$$
\operatorname{dim} H^{1}\left(G, H^{1}\left(X(\mathbb{C}), \mathbb{Z}_{-}\right)\right) \leqslant q(X),
$$

причем для $M$-поверхности это неравенство становится равенством. Первое утверждение теоремы доказано, перейдем к доказательству второго. Из точной последовательности (16) получаем, что в условиях этого утверждения группа $H_{\mathrm{alg}}^{2}\left(X(\mathbb{C}) ; G, \mathbb{Z}_{-}\right)$ равна $\mathbb{Z}^{\rho(X)}$. Пусть $X$ является $(M-k)$-поверхностью, а $\varkappa$-размерностью ядра гомоморфизма

$$
\beta: H_{\text {alg }}^{2}\left(X(\mathbb{C}) ; G, \mathbb{Z}_{-}\right) \otimes \mathbb{F}_{2} \rightarrow H^{1}\left(X(\mathbb{R}), \mathbb{F}_{2}\right) .
$$

Тогда для доказательства неравенств (12) достаточно установить, что $\varkappa \leqslant k$. Рассмотрим точную последовательность групп

$$
0 \rightarrow H_{\mathrm{alg}}^{2}\left(X(\mathbb{C}) ; G, \mathbb{Z}_{-}\right) \rightarrow H^{2}\left(X(\mathbb{C}) ; G, \mathbb{Z}_{-}\right) \rightarrow H_{\mathrm{tr}}^{2}\left(X(\mathbb{C}) ; G, \mathbb{Z}_{-}\right) \rightarrow 0 .
$$

Так как $H_{\operatorname{tr}}^{2}\left(X(\mathbb{C}) ; G, \mathbb{Z}_{-}\right)=H^{2}\left(X(\mathbb{C}) ; G, \mathbb{Z}_{-}\right) / \delta H^{1}\left(X(\mathbb{C}) ; G, \mathscr{O}^{*}\right)$, из точной последовательности (16) следует, что всегда группа $H_{\mathrm{tr}}^{2}(X(\mathbb{C}) ; G, \mathbb{Z}-)$ свободная. Следовательно, из точной последовательности (18) получаем точную последовательность $\mathbb{F}_{2}$-пространств

$0 \rightarrow H_{\mathrm{alg}}^{2}\left(X(\mathbb{C}) ; G, \mathbb{Z}_{-}\right) \otimes \mathbb{F}_{2} \rightarrow H^{2}\left(X(\mathbb{C}) ; G, \mathbb{Z}_{-}\right) \otimes \mathbb{F}_{2} \rightarrow H_{\mathrm{tr}}^{2}\left(X(\mathbb{C}) ; G, \mathbb{Z}_{-}\right) \otimes \mathbb{F}_{2} \rightarrow 0$

Отсюда следует, что размерность ядра гомоморфизма (17) не превосходит размерности ядра гомоморфизма (14). Чтобы закончить доказательство неравенств (12), достаточно установить, что размерность ядра гомоморфизма (14) равна $k$. Из спектральной последовательности $\mathrm{II}(X(\mathbb{C}) ; G, \mathbb{Z}-)$ следует, что в условиях второго утверждения гомоморфизм

$$
\alpha: H^{2}\left(X(\mathbb{C}) ; G, \mathbb{Z}_{-}\right) \rightarrow H^{2}\left(X(\mathbb{C}), \mathbb{Z}_{-}\right)^{G}
$$

является изоморфизмом. В работах [4], [5] показано, что элемент $h \in H^{2}\left(X(\mathbb{C}) ; G, \mathbb{Z}_{-}\right)$ принадлежит $\operatorname{Ker} \beta$ тогда и только тогда, когда $\alpha(h) \in\left(1+g^{*}\right) H^{2}\left(X(\mathbb{C}), \mathbb{Z}_{-}\right)$. Таким образом, размерность ядра гомоморфизма (14) равна размерности подпространства $\left(1+g^{*}\right) H^{2}\left(X(\mathbb{C}), \mathbb{F}_{2}\right)$ пространства $H^{2}\left(X(\mathbb{C}), \mathbb{Z}_{-}\right){ }^{G} \otimes \mathbb{F}_{2}$. Из равенств (см. [6])

$$
\begin{gathered}
\operatorname{dim} H^{*}\left(X(\mathbb{R}), \mathbb{F}_{2}\right)=2+\operatorname{dim} H^{2}\left(X(\mathbb{C}), \mathbb{F}_{2}\right)-2 k \\
\operatorname{dim} H^{*}\left(X(\mathbb{R}), \mathbb{F}_{2}\right)=2+\operatorname{dim} H^{1}\left(G, H^{2}\left(X(\mathbb{C}), \mathbb{F}_{2}\right)\right)
\end{gathered}
$$


следует, что $\operatorname{dim}\left(1+g^{*}\right) H^{2}\left(X(\mathbb{C}), \mathbb{F}_{2}\right)=k$. Итак, неравенства $(12)$ теоремы доказаны. Неравенства (13) следуют из неравенств (12) и равенств

$$
\begin{gathered}
\rho_{0}(X)=\operatorname{rk} H^{2}\left(X(\mathbb{C}), \mathbb{Z}_{-}\right)^{G}-\rho(X), \quad \rho_{0}(X(\mathbb{R}))=\operatorname{dim} H^{1}\left(X(\mathbb{R}), \mathbb{F}_{2}\right)-\rho(X(\mathbb{R})), \\
\operatorname{dim} H^{1}\left(X(\mathbb{R}), \mathbb{F}_{2}\right)=\operatorname{rk} H^{2}\left(X(\mathbb{C}), \mathbb{Z}_{-}\right)^{G}-k
\end{gathered}
$$

Равенства (19) вытекают из определения чисел Лефшеца, а (20) установлено в [6]. Теорема доказана.

\section{СПИСОК ЦИТИРОВАННОЙ ЛИТЕРАТУРЫ}

[1] Краснов В. А. Характеристические классы векторных расслоений на вещественном алгебраическом многообразии // Изв. АН СССР. Сер. матем. 1991. Т. 55. № 4. С. 716-746.

[2] Краснов В.А. Когомологическая группа Брауэра вещественного алгебраического многообразия // Изв. РАН. Сер. матем. 1996. Т. 60. № 5. С. 57-88.

[3] Харламов В. М. Обобщенное неравенство Петровского // Функцион. анализ и его прилож. 1974. T. 8. № 2. C. 50-56.

[4] Краснов В.А. Об эквивариантных когомологиях Гротендика вещественного алгебраического многообразия и их приложениях // Изв. РАН. Сер. матем. 1994. Т. 58. № 3. С. 36-52.

[5] К раснов В.А. Алгебраические циклы на вещественном алгебраическом $G M$-многообразии и их приложения // Изв. РАН. Сер. матем. 1993. Т. 57. № 4. С. 153-173.

[6] Краснов В.А. Неравенства Гарнака-Тома для отображений вещественных алгебраических многообразий // Изв. АН СССР. Сер. матем. 1983. Т. 47. № 2. С. 268-297.

Ярославский государственньй университет

Поступило 27.01.97 